\title{
Características del síndrome post cuidado intensivo: revisión de alcance*
}

\author{
Characteristic of post ICU syndrome: a review of scope \\ Características da síndrome pós-cuidados intensivos: revisão do escopo
}

Ángela Maria Henao-Castaño ${ }^{\text {a }}$

Universidad Nacional de Colombia, Colombia

DOI: https://doi.org/10.11144/Javeriana.ie23.csci

angmhenaocas@unal.edu.co

ORCID: https://orcid.org/0000-0003-4203-0016

Recibido: 04 Enero 2021

Angie Vanessa Villamil Buitrago

Aceptado: 07 Octubre 2021

Universidad Nacional de Colombia, Colombia

Publicado: 30 Diciembre 2021

ORCID: https://orcid.org/0000-0002-4582-3989

Sebastián Marin Ramírez

Universidad Nacional de Colombia, Colombia

ORCID: https://orcid.org/0000-0002-8152-405

Camila Andrea Cogollo Hernández

Universidad Nacional de Colombia, Colombia

ORCID: https://orcid.org/0000-0002-6415-503

\section{Resumen:}

Objetivo: identificar la evidencia disponible de las características del síndrome post unidad de cuidado intensivo en el paciente que egresa de la unidad de cuidado intensivo. Método: revisión de alcance con la metodología del Joanna Briggs Institut, en cinco bases de datos con la estrategia de búsqueda ([PostIntensive AND Care AND Syndrome] [Nursing AND Care AND PICS]). Se incluyeron estudios primarios publicados entre los años 2010 y 2020, disponibles en texto completo. Resultados: después del análisis de los 48 estudios primarios se extrajeron seis categorías temáticas así: aspecto cognitivo en el síndrome post UCI, osteomusculares, psicoemocionales, estrategias de prevención con modelos de sobrevivientes de UCI, síndrome post unidad cuidado intensivo en la familia y herramientas de evaluación. Conclusiones: mejorar la calidad de atención de los pacientes que presentan este síndrome, prevenir la aparición y aumentar la calidad de vida prestada a los sobrevivientes de UCI y sus familias. La evidencia reporta que entre los cuidadores se pueden desencadenar distintos trastornos que disminuyen su calidad de vida. La enfermería juega un papel crucial en la prevención de la aparición del síndrome mediante estrategias en la UCI e interdisciplinares; los planes de egreso y seguimiento a los pacientes pueden lograr que estos se rehabiliten más rápido y eviten discapacidades o secuelas a largo plazo.

Palabras clave: síndrome post cuidados intensivos, cuidados críticos, delirium, enfermedad crítica.

\section{Abstract:}

Objective: to identify the available evidence regarding the post ICU syndrome in patients leaving the intensive care unit. Methods: it is a review of scope according to the Joanna Briggs Institut methodology, in 5 data bases with the search strategy ([PostIntensive AND Care AND Syndrome] [Nursing AND Care AND PICS]). Primary studies published between 2010 and 2020 with full text available were included. Results: having analyzed 48 primary studies, 6 thematic categories were created: cognitive aspect in post ICU syndrome, musculoskeletal, psycho-emotional, prevention strategies with models of ICU survivors, post ICU syndrome in the family and evaluation tools. Conclusions: To improve the health care in patient in post ICU syndrome, to prevent the syndrome emergence and increase the quality of life provided to the ICU survivors and their families. Evidence shows that caregivers can develop different disorders that impact their quality of life. Nursing plays a crucial role in preventing the syndrome advent by using interdisciplinary and ICU strategies. This charge and follow-up plans can make these patients to achieve a faster rehabilitation and avoid impairment or sequels in the future.

Keywords: post ICU syndrome, critical care, delirium, critical disease.

Notas de autor

\footnotetext{
a Autora de correspondencia: angmhenaocas@unal.edu.co
} 


\section{Resumo:}

Objetivo: identificar a evidência disponível das características da síndrome pós-unidade de terapia intensiva em pacientes egressos da unidade de terapia intensiva. Método: revisão do escopo com a metodologia do Joanna Briggs Institut, em cinco bases de dados com a estratégia de busca ([PostIntensive AND Care AND Syndrome] [Nursing AND Care AND PICS]). Incluíram-se estudos primários publicados entre 2010 e 2020, disponíveis em texto completo. Resultados: após análise dos 48 estudos primários foram extraídas seis categorias temáticas: aspecto cognitivo na síndrome pós-UTI, osteomuscular, psicoemocional, estratégias de prevenção com modelos de sobrevivência na UTI, síndrome pós-unidade de Terapia intensiva na família e ferramentas de avaliação. Conclusões: melhorar a qualidade da atenção dos pacientes que apresentam a síndrome, prevenir a aparição e aumentar a qualidade de vida prestada aos sobreviventes da UTI e suas famílias. Evidências relatam que diversos transtornos podem se desencadear entre os cuidadores que reduzem sua qualidade de vida. A enfermagem tem um papel crucial na prevenção da aparição da síndrome mediante estratégias na UTI e interdisciplinares; planos de alta e acompanhamento aos pacientes podem conseguir uma reabilitação mais rápida e evitar incapacidades ou sequelas no longo prazo.

Palavras-chave: síndrome pós-cuidados intensivos, cuidados críticos, delirium, doença crítica.

\section{Introducción}

En el transcurso de la vida, las personas pueden adquirir una enfermedad crítica. Cuando esta se encuentra en un contexto que propicie su complicación, así sucederá, y es allí donde, para tratar de salvar las vidas de quienes padecen estas enfermedades, se ingresa en la unidad de cuidados intensivos; lugar en el que se realizan diversos y complejos procedimientos. Los avances en la medicina han logrado aumentar la población de supervivientes de la Unidad de Cuidado Intensivo (UCI); sin embargo, puede presentarse el síndrome post UCI (PICS por sus siglas en inglés), relacionado con las deficiencias físicas, cognitivas y mentales que ocurren durante la estadía en la UCI, después del alta de esta o del hospital, y vinculada también con el pronóstico a largo plazo de los pacientes de UCI $(1,2)$. El síndrome se trata de forma multidisciplinaria y es común encontrar literatura con abordajes desde la medicina, la terapia física y la enfermería, entre otras áreas $(3,4)$. Así, es notable que la enfermería, al ser una profesión en contacto constante con los pacientes, recibe llamados para realizar intervenciones, ya sea en pro de disminuir la presencia de este síndrome en las personas o para prevenir desde la UCI su aparición (5). Por lo tanto, para lograr prevenirlo y tratarlo es de gran importancia conocer las características que lo acompañan.

No solo los pacientes se ven afectados por la presencia de este síndrome, también su familia, ya que cuando el enfermo pierde alguna de sus capacidades, y resulta impedido para el desarrollo de una vida normal, es común que los familiares se hagan cargo, asuman la labor de cuidadores y encuentren parte de su vida ocupada por la carga que esta dedicación supone. Esta situación reduce sus horas de trabajo y afecta su economía; incluso se ven obligados a retirarse de sus trabajos y a pasar por situaciones complejas. El objetivo de esta revisión fue identificar las características del PICS en el paciente que egresa de la unidad de cuidado intensivo.

\section{Método}

La scopingreview se llevó a cabo siguiendo las etapas propuestas por la metodología del Joanna Briggs Institute (6). Pregunta para la revisión. Para realizar esta pregunta se tuvieron en cuenta las directrices planteadas con la estrategia Población, Concepto, Contexto, PPC, y se formuló de la siguiente manera: ¿cuál es la evidencia disponible de las características del síndrome post unidad de cuidado intensivo en el paciente que egresa de la UCI? Criterios de inclusión y exclusión. Población: esta revisión consideró estudios centrados en pacientes adultos que sobrevivieron a la unidad de cuidado intensivo. Concepto: esta revisión abordó los estudios que incluyen las diferentes características del síndrome post cuidado intensivo en sobrevivientes de la enfermedad crítica. Contexto: la revisión incluyó estudios desarrollados en unidad de cuidado intensivo médica de adulto, unidad de cuidado paliativo y unidad de cuidado posquirúrgico. 
Los estudios incluidos contaron con el acceso a texto completo sin restricción en el idioma, a resultados de estudios originales de tipo cualitativo, cuantitativo y a artículos de revisión publicados en el período comprendido entre enero de 2010 y noviembre de 2020, con un nivel de evidencia medio a alto; por lo cual se excluyeron artículos de opinión o cartas al editor, libros y literatura gris, así como aquellos donde el contexto omitía la unidad de cuidado intensivo adulto. Estrategia de búsqueda. Se consultaron cinco bases, Tripdatabase, Scopus, Biblioteca Virtual de Salud, Scielo y ScienceDirect, donde se hallaron artículos de los últimos 10 años, que cumplieran con las ecuaciones de búsqueda utilizadas para cada una de bases: PostIntensive AND Care AND Syndrome; Nursing AND Care AND PICS; PICS AND Treatment AND Nurse; Síndrome Post Unidad de Cuidados Intensivos AND Enfermería; PostIntensive Care Syndrome OR Post-Intensive Care Syndrome OR Post-ICU Syndrome AND Nursing. Extracción de los datos. Los datos se tomaron de los estudios incluidos en la revisión, por dos revisores de manera independiente, usando la herramienta estándar de extracción de datos del JBI para revisión cualitativa. La información extraída incluía población, contexto, conceptos, y el análisis se llevó a cabo por medio de una matriz en Excel, con los 48 artículos incluidos, que identificó de manera individual autores, año, idioma, país, tipo de estudio y tamaño de muestra. Asimismo, se evaluaron cado uno de los estudios con el instrumento Caspe (7), para calificar la validez, con un valor por encima de siete por dos evaluadores. En los casos donde se tenían desacuerdos se contó con un tercer evaluador.

\section{Resultados}

La búsqueda en diferentes bases de datos produjo un total de 752 referencias; después de eliminar los artículos duplicados, de reflexión y de revisión, resultaron 56 artículos, cuyos títulos y resúmenes fueron evaluados por los revisores, quienes leyeron los textos completos para evaluar su calidad, extraer datos y determinar su inclusión. Finalmente se seleccionaron 48 estudios de las bases de datos para esta revisión, figura 1. 


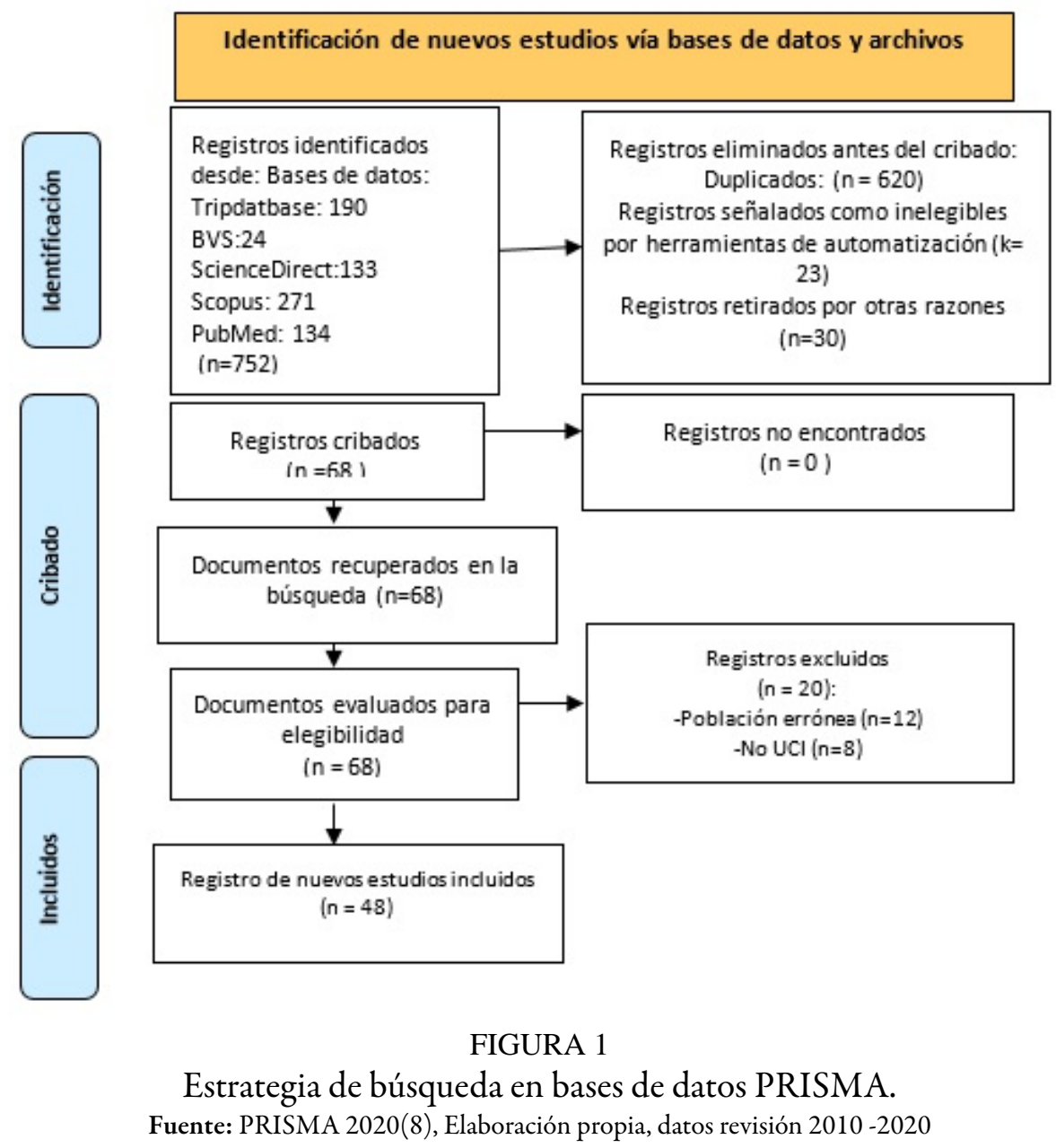

Se realizó una revisión general de los artículos incluidos: $29 \%$ fueron estudios publicados en 2019; en 2018, 23 \%; en 2017,17 \%, y para 2020, 17 \%; los años 2010, 2015 y 2016 arrojaron el 4 \% cada uno. El principal idioma de publicación fue el inglés con $85 \%$; mientras que el español y portugués obtuvieron 8 $\%$ y $7 \%$ respectivamente. Los porcentajes de los artículos por regiones y países de origen fueron: $31 \%$ para Estados Unidos; Europa $25 \%$; Países Bajos $21 \%$; Australia $8 \%$; Asia $6 \%$, y Brasil; Argentina; Canadá e Irán $2 \%$ cada uno. La población de estudio estuvo comprendida por una media de 48 pacientes y 125 cuidadores, como lo presenta la tabla 1 . Los estudios incluidos con metodologías cualitativa y cuantitativa aportan a los diferentes temas presentes en la tabla 2. 
TABLA 1

Distribución de los artículos según temáticas y metodología.

\begin{tabular}{|c|c|c|c|c|c|}
\hline Tema & Contenidos & $\begin{array}{l}\text { Cualitativo } \\
\mathrm{s}\end{array}$ & $\begin{array}{l}\text { Observacionale } \\
\mathrm{s}\end{array}$ & $\begin{array}{l}\text { Experimental } \\
\text { es }\end{array}$ & Total (\%) \\
\hline $\begin{array}{l}\text { Estrategias de } \\
\text { prevención } \\
\text { con Modelos } \\
\text { de } \\
\text { sobreviviente } \\
\text { s de UCI }\end{array}$ & $\begin{array}{l}\text { Conciliación de } \\
\text { medicamentos, } \\
\text { ambiente de la } \\
\text { UCI, } \\
\text { Rehabilitación } \\
\text { temprana. }\end{array}$ & 2 & 10 & 2 & $14(29)$ \\
\hline PICS Familia & $\begin{array}{l}\text { Ansiedad, } \\
\text { depresión, } \\
\text { alteración sueño, }\end{array}$ & 2 & 5 & 2 & $9(19)$ \\
\hline $\begin{array}{l}\text { Aspectos } \\
\text { cognitivos }\end{array}$ & $\begin{array}{l}\text { Pérdida de } \\
\text { memoria, } \\
\text { problemas en } \\
\text { concentración }\end{array}$ & 0 & 5 & 2 & $7(15)$ \\
\hline $\begin{array}{l}\text { Aspectos } \\
\text { Osteomuscula } \\
\text { res }\end{array}$ & $\begin{array}{l}\text { Presencia de } \\
\text { fatiga, disnea, } \\
\text { debilidad y } \\
\text { dolor }\end{array}$ & 1 & 3 & 1 & $5(10)$ \\
\hline $\begin{array}{l}\text { Aspectos } \\
\text { Psicoemocion } \\
\text { ales }\end{array}$ & $\begin{array}{l}\text { Disfunción } \\
\text { ejecutiva, } \\
\text { depresión }\end{array}$ & 0 & 6 & 2 & $8(17)$ \\
\hline $\begin{array}{l}\text { Herramientas } \\
\text { para evaluar } \\
\text { PICS }\end{array}$ & $\begin{array}{l}\text { Escalas para } \\
\text { medir ansiedad, } \\
\text { depresión, } \\
\text { calidad de vida. }\end{array}$ & 0 & 4 & 1 & $5(10)$ \\
\hline Total & & 5 & 33 & 10 & $48(100)$ \\
\hline
\end{tabular}

Fuente: Elaboración propia, datos revisión 2010 -2020

TABLA 2

Artículos incluidos en la revisión y nivel de evidencia

\begin{tabular}{|c|c|c|c|c|c|c|}
\hline Diseño & $\begin{array}{l}\text { Casp } \\
\text { e }\end{array}$ & Autores & Año & País & $\begin{array}{l}\text { Muestra y } \\
\text { Población }\end{array}$ & JBI \\
\hline $\begin{array}{l}\text { Descriptivo } \\
\text { Cualitativo }\end{array}$ & 9 & $\begin{array}{l}\text { Sabine Adriana Johanna } \\
\text { Josepha, Maaike et al. }\end{array}$ & 2020 & Holanda & $\begin{array}{l}13 \\
\text { pacientes }\end{array}$ & $\begin{array}{l}\text { Nivel } 4 \\
\text { Grado } \\
\text { B }\end{array}$ \\
\hline $\begin{array}{l}\text { Descriptivo, } \\
\text { Longitudinal, } \\
\text { Multicéntrico, } \\
\text { Prospectivo }\end{array}$ & 8 & $\begin{array}{l}\text { Amy B. Petrinec, Bradley R. } \\
\text { Martin }\end{array}$ & 2017 & $\begin{array}{l}\text { Inglaterr } \\
\text { a }\end{array}$ & $\begin{array}{l}68 \\
\text { pacientes } \\
\text { y } \\
\text { familiares }\end{array}$ & $\begin{array}{l}\text { Nivel } \\
\text { 3.e. } \\
\text { Grado } \\
\text { B }\end{array}$ \\
\hline $\begin{array}{l}\text { Cohorte, } \\
\text { Prospectivo }\end{array}$ & 8 & $\begin{array}{l}\text { Annachiara Marra, Pratik P. } \\
\text { Pandharipande, Timothy D. et } \\
\text { al. }\end{array}$ & 2018 & USA & $\begin{array}{l}465 \\
\text { pacientes }\end{array}$ & $\begin{array}{l}\text { Nivel } \\
\text { 3.e. } \\
\text { Grado } \\
\text { B }\end{array}$ \\
\hline $\begin{array}{l}\text { Etnografia } \\
\text { focalizada }\end{array}$ & 8 & $\begin{array}{l}\text { Ann Louise Bodker Hanifa, } \\
\text { Anne Okkels Glaemose, et al. }\end{array}$ & 2018 & $\begin{array}{l}\text { Dinamar } \\
\text { ca }\end{array}$ & $\begin{array}{l}10 \\
\text { pacientes } \mathrm{C}\end{array}$ & $\begin{array}{l}\text { Nivel } 4 \\
\text { Grado } \\
\text { B }\end{array}$ \\
\hline $\begin{array}{l}\text { Estudio de } \\
\text { Viabilidad } \\
\text { Prospectivo y } \\
\text { Observacional }\end{array}$ & 8 & $\begin{array}{l}\text { Carla M. Sevin, Sarah L. } \\
\text { Bloom, James C. Jackson, Li } \\
\text { Wang, E.Wesley Ely, Joanna } \\
\text { L. Stollings }\end{array}$ & 2018 & USA & $\begin{array}{l}218 \\
\text { pacientes }\end{array}$ & $\begin{array}{l}\text { Nivel } \\
\text { 3.e. } \\
\text { Grado } \\
\text { B }\end{array}$ \\
\hline $\begin{array}{l}\text { Estudio de } \\
\text { Cohorte } \\
\text { Retrospectivo }\end{array}$ & 9 & $\begin{array}{l}\text { Ilse van Beusekom, Ferishta } \\
\text { Bakhshi-Raiez, Nicolette F. et } \\
\text { al. }\end{array}$ & 2019 & Holanda & $\begin{array}{l}\text { Historia } \\
\text { clinica } \\
2013-2014\end{array}$ & $\begin{array}{l}\text { Nivel } \\
\text { 3.e. } \\
\text { Grado } \\
\text { B }\end{array}$ \\
\hline $\begin{array}{l}\text { Estudio } \\
\text { Prospectivo } \\
\text { Observacional }\end{array}$ & 8 & $\begin{array}{l}\text { J. Torres, D. Carvalhoa, E. } \\
\text { Molinosa, C. Valesa, A. } \\
\text { Ferreira, C.C. Dias, R. Araújo, } \\
\text { E. Gomes }\end{array}$ & 2017 & España & $\begin{array}{l}245 \\
\text { pacientes }\end{array}$ & $\begin{array}{l}\text { Nivel } \\
\text { 3.e. } \\
\text { Grado } \\
\text { B } \\
\end{array}$ \\
\hline $\begin{array}{l}\text { Teoría } \\
\text { Fundamentada }\end{array}$ & 9 & Jiyeon Kang, Yeon Jin Jeong & 2018 & Corea & $\begin{array}{l}13 \\
\text { sobrevivie } \\
\text { ntes }\end{array}$ & $\begin{array}{l}\text { Nivel } 4 \\
\text { Grado } \\
\text { B }\end{array}$ \\
\hline $\begin{array}{l}\text { Estudio de } \\
\text { Cohorte } \\
\text { Prospectivo } \\
\text { Observacional }\end{array}$ & 8 & $\begin{array}{l}\text { Joanna L. Stollings, Sarah L. } \\
\text { Bloom, Li Wang, E. Wesley } \\
\text { Ely, James C. Jackson, and } \\
\text { Carla M. Sevin }\end{array}$ & 2018 & USA & $\begin{array}{l}56 \\
\text { pacientes }\end{array}$ & $\begin{array}{l}\text { Nivel } \\
\text { 3.e. } \\
\text { Grado } \\
\text { B }\end{array}$ \\
\hline $\begin{array}{l}\text { Es un estudio } \\
\text { de cohorte } \\
\text { transversal, } \\
\text { retrospectivo y } \\
\text { multicéntrico }\end{array}$ & 8 & $\begin{array}{l}\text { Johan H. Vlake, Michel E. van } \\
\text { Genderen, Anna Schut, Martijn } \\
\text { Verkade, Evert-Jan Wils, et al }\end{array}$ & 2020 & Holanda & $\begin{array}{l}44 \\
\text { pacientes }\end{array}$ & $\begin{array}{l}\text { Nivel } \\
4 \mathrm{~b} . \\
\text { Grado } \\
B\end{array}$ \\
\hline $\begin{array}{l}\text { Estudio de } \\
\text { Cohorte }\end{array}$ & 8 & $\begin{array}{l}\text { Monika C. Kerckhoffs, Felicia } \\
\text { F. L. Kosasi, Ivo W. Soliman, } \\
\text { et al. }\end{array}$ & 2019 & Holanda & $\begin{array}{l}1453 \\
\text { pacientes }\end{array}$ & $\begin{array}{l}\text { Nivel } 4 \\
\text { Grado } \\
\text { B }\end{array}$ \\
\hline Cohorte & 8 & $\begin{array}{l}\text { By Sophia Wang, Duane Allen, } \\
\text { Anthony Perkins, Patrick } \\
\text { Monahan, Sikandar Khan, Sue }\end{array}$ & 2019 & USA & $\begin{array}{l}142 \\
\text { pacientes }\end{array}$ & $\begin{array}{l}\text { Nivel } 4 \\
\text { Grado } \\
\text { B }\end{array}$ \\
\hline
\end{tabular}




\begin{tabular}{|c|c|c|c|c|c|c|}
\hline & & $\begin{array}{l}\text { Lasiter, Malaz Boustani and } \\
\text { Babar Khan }\end{array}$ & & & & \\
\hline $\begin{array}{l}\text { E1 estudio fue } \\
\text { una encuesta } \\
\text { transversal de } \\
\text { un solo centro }\end{array}$ & 8 & $\begin{array}{l}\text { Sumeet Rai, Lakmali Anthony, } \\
\text { Dale M. Needham, Et al. }\end{array}$ & 2019 & Australia & $\begin{array}{l}198 \text { stafff } \\
\text { UCI }\end{array}$ & \begin{tabular}{|l|} 
Nivel \\
$4 \mathrm{~b}$. \\
Grado \\
$\mathrm{B}$ \\
\end{tabular} \\
\hline $\begin{array}{l}\text { Estudio } \\
\text { transversal }\end{array}$ & 8 & $\begin{array}{l}\text { Juliana Bessa Martins ,Adriana } \\
\text { Alves dos Santos, Et al. }\end{array}$ & 2019 & Brasil & $\begin{array}{l}335 \\
\text { pacientes }\end{array}$ & \begin{tabular}{|l|} 
Nivel \\
$3 \mathrm{c}$. \\
Grado \\
B \\
\end{tabular} \\
\hline $\begin{array}{l}\text { Estudio de } \\
\text { cohorte }\end{array}$ & 8 & $\begin{array}{l}\text { Michael D. Wood, David M. } \\
\text { Maslove, John Muscedere, } \\
\text { Stephen H. Scott, J. Gordon } \\
\text { Boyd. }\end{array}$ & 2018 & Canadá & \begin{tabular}{|l|}
70 \\
pacientes
\end{tabular} & \begin{tabular}{|l|} 
Nivel \\
3c. \\
Grado \\
B \\
\end{tabular} \\
\hline $\begin{array}{l}\text { Estudio } \\
\text { transversal }\end{array}$ & 8 & \begin{tabular}{|l|} 
Volha Dziadzko, Mikhail A. \\
Dziadzko, Margaret M. \\
Johnson, Ognjen Gajic, \\
Lioudmila V. Karnatovskaia \\
\end{tabular} & 2017 & USA & \begin{tabular}{|l}
50 \\
pacientes \\
44 \\
familias \\
\end{tabular} & $4 . b$ \\
\hline $\begin{array}{l}\text { Estudio de } \\
\text { cohorte }\end{array}$ & 9 & $\begin{array}{l}\text { Alice Chiarici, Elisa } \\
\text { Andrenelli, Oletta Serpilli, } \\
\text { Matteo Andreolini, et al. }\end{array}$ & 2019 & Italia & \begin{tabular}{|l|l|}
285 \\
pacientes
\end{tabular} & \begin{tabular}{|l|} 
Nivel \\
$3 \mathrm{c}$. \\
Grado \\
B \\
\end{tabular} \\
\hline $\begin{array}{l}\text { Estudio } \\
\text { transversal }\end{array}$ & 8 & $\begin{array}{l}\text { Margo van Mol, Erwin Ista, } \\
\text { Monique van Dijk }\end{array}$ & 2018 & \begin{tabular}{|l|} 
Paises \\
Bajos
\end{tabular} & \begin{tabular}{|l|}
140 \\
enfermera \\
$\mathrm{s}$ \\
\end{tabular} & 4.b \\
\hline $\begin{array}{l}\text { Estudio de } \\
\text { cohorte }\end{array}$ & 8 & $\begin{array}{l}\text { Lioudmila V. Karnatovskaia, } \\
\text { Phillip J. Schulte, et al. }\end{array}$ & 2019 & USA & \begin{tabular}{|l|}
155 \\
pacientes
\end{tabular} & \begin{tabular}{|l|} 
Nivel \\
$3 c$. \\
Grado \\
B \\
\end{tabular} \\
\hline $\begin{array}{l}\text { Estudio de } \\
\text { cohorte }\end{array}$ & 9 & $\begin{array}{l}\text { Georgios Sidiras, Irini Patsaki, } \\
\text { Eleftherios Karatzanos, Maria } \\
\text { Dakoutrou, et al. }\end{array}$ & 2019 & Grecia & \begin{tabular}{|l|}
128 \\
pacientes
\end{tabular} & \begin{tabular}{|l|} 
Nivel \\
3c. \\
Grado \\
B \\
\end{tabular} \\
\hline $\begin{array}{l}\text { Estudio de } \\
\text { cohorte }\end{array}$ & 8 & $\begin{array}{l}\text { JiYeon Choi, Judith A. Tate, } \\
\text { Michael P. Donahoe, Dianxu } \\
\text { Renb, Leslie A. Hoffman, } \\
\text { Eileen R. Chasens } \\
\end{array}$ & 2016 & \begin{tabular}{|l} 
Estados \\
Unidos
\end{tabular} & \begin{tabular}{|l|}
11 \\
familias
\end{tabular} & \begin{tabular}{|l|} 
Nivel \\
3c. \\
Grado \\
B \\
\end{tabular} \\
\hline $\begin{array}{l}\text { Estudio de } \\
\text { cohorte }\end{array}$ & 8 & $\begin{array}{l}\text { Chrystal L. Lewis, Jessica Z. } \\
\text { Taylor }\end{array}$ & 2017 & $\begin{array}{l}\text { Estados } \\
\text { Unidos }\end{array}$ & $\begin{array}{l}127 \\
\text { familias }\end{array}$ & \begin{tabular}{|l|} 
Nivel \\
3c. \\
Grado \\
B
\end{tabular} \\
\hline $\begin{array}{l}\text { Estudio de } \\
\text { cohorte }\end{array}$ & 8 & $\begin{array}{l}\text { Nathan Ferrand, Cédrick } \\
\text { Zaouter, Brigitte Chastel, } \\
\text { Karim Faye, et al. }\end{array}$ & 2019 & Francia & \begin{tabular}{|l|}
160 \\
participant \\
es
\end{tabular} & \begin{tabular}{|l|} 
Nivel \\
3c. \\
Grado \\
B \\
\end{tabular} \\
\hline $\begin{array}{l}\text { Cohorte } \\
\text { retrospectiva }\end{array}$ & 8 & $\begin{array}{l}\text { Yu Jin Seo, Sae Rom Park, } \\
\text { Jung Hoon Lee, Chul Jung, et } \\
\text { al. } \\
\end{array}$ & 2020 & \begin{tabular}{|l|} 
Repúblic \\
a de \\
Corea \\
\end{tabular} & \begin{tabular}{|l|}
121 \\
pacientes
\end{tabular} & $3 . e$ \\
\hline $\begin{array}{l}\text { Estudio de } \\
\text { cohorte }\end{array}$ & 8 & $\begin{array}{l}\text { Debbie G.A. Janssen, Joep } \\
\text { Jonker, Diederik van Dijk, et } \\
\text { al. }\end{array}$ & 2020 & $\begin{array}{l}\text { Paises } \\
\text { Bajos }\end{array}$ & \begin{tabular}{|l|}
1328 \\
sobrevivie \\
ntes
\end{tabular} & \begin{tabular}{|l|} 
Nivel \\
$3 \mathrm{c}$. \\
Grado \\
B \\
\end{tabular} \\
\hline $\begin{array}{l}\text { Estudio de } \\
\text { cohorte }\end{array}$ & 7 & $\begin{array}{l}\text { Carol L. Hodgson, Kimberley } \\
\text { J. Haines, Michael Bailey, } \\
\text { Jonathan Barrett, Rinaldo } \\
\text { Bellomo, Tracey Bucknall, et } \\
\text { al }\end{array}$ & 2018 & Australia & \begin{tabular}{|l|}
373 \\
pacientes
\end{tabular} & $\begin{array}{l}\text { Nivel } \\
\text { 3c. } \\
\text { Grado } \\
\text { B }\end{array}$ \\
\hline $\begin{array}{l}\text { Estudio de } \\
\text { cohorte }\end{array}$ & 7 & $\begin{array}{l}\text { Jenny Rosendahl, Hristina } \\
\text { Kisyova, Romina Gawlytta, } \\
\text { André Scherag }\end{array}$ & 2019 & $\begin{array}{l}\text { Alemani } \\
\text { a }\end{array}$ & \begin{tabular}{|l|}
110 \\
pacientes
\end{tabular} & \begin{tabular}{|l|} 
Nivel \\
3c. \\
Grado \\
B \\
\end{tabular} \\
\hline $\begin{array}{l}\text { Cohorte } \\
\text { retrospectiva }\end{array}$ & 8 & \begin{tabular}{|l|} 
Ilse van Beusekom, Ferishta \\
Bakhshi-Raiez, Marike van der \\
Schaaf, Dave A. et al.
\end{tabular} & 2020 & $\begin{array}{l}\text { Paises } \\
\text { Bajos }\end{array}$ & \begin{tabular}{|l|}
49,004 \\
pacientes
\end{tabular} & \begin{tabular}{|l|} 
Nivel \\
3c. \\
Grado \\
B \\
\end{tabular} \\
\hline Pragmático & 7 & $\begin{array}{l}\text { Oystein Tronstad, Dylan } \\
\text { Flaws, India Lye, John F. } \\
\text { Fraser, Sue Patterson. }\end{array}$ & 2020 & Australia & \begin{tabular}{|l|}
30 \\
médicos y \\
enfermero \\
$\mathrm{s}$ \\
\end{tabular} & \begin{tabular}{|l|} 
Nivel \\
3c. \\
Grado \\
B \\
\end{tabular} \\
\hline $\begin{array}{l}\text { Estudio de } \\
\text { cohorte }\end{array}$ & 6 & \begin{tabular}{|l|} 
Michael E. Detsky, Rachel \\
Kohn, Aaron M. Delman, Anna \\
E. Buehler, Saida A. Kent, \\
Isabella V. et al \\
\end{tabular} & 2018 & USA & \begin{tabular}{|l|}
303 \\
pacientes
\end{tabular} & \begin{tabular}{|l|} 
Nivel \\
3c. \\
Grado \\
B \\
\end{tabular} \\
\hline $\begin{array}{l}\text { Estudio de } \\
\text { cohorte }\end{array}$ & 8 & $\begin{array}{l}\text { Lynda M. Thurston, Sharyn L. } \\
\text { Milnes, Carol L. Hodgson, } \\
\text { Danielle E. Berkovic, et al. }\end{array}$ & 2020 & \begin{tabular}{|l|} 
Australia \\
$\mathrm{y}$ \\
Estados \\
Unidos \\
\end{tabular} & $\begin{array}{l}89 \\
\text { pacientes }\end{array}$ & \begin{tabular}{|l|} 
Nivel \\
3c. \\
Grado \\
B \\
\end{tabular} \\
\hline $\begin{array}{l}\text { Estudio de } \\
\text { cohorte }\end{array}$ & 7 & \begin{tabular}{|l} 
Andrea V. Das Neves, Daniela \\
N. Vasquez, Cecilia I. Loudet, \\
Dante Intile, Maria Gabriela \\
Sáenz, et al. \\
\end{tabular} & 2015 & $\begin{array}{l}\text { Argentin } \\
\text { a }\end{array}$ & \begin{tabular}{|l|}
112 \\
pacientes
\end{tabular} & \begin{tabular}{|l|} 
Nivel \\
3c. \\
Grado \\
B \\
\end{tabular} \\
\hline $\begin{array}{l}\text { Ensayo clínico } \\
\text { aleatorizado }\end{array}$ & 9 & $\begin{array}{l}\text { Irini Patsaki, Vasiliki } \\
\text { Gerovasili, Georgios Sidiras, } \\
\text { Eleftherios Karatzanos, et al }\end{array}$ & 2017 & Grecia & \begin{tabular}{|l|}
128 \\
pacientes
\end{tabular} & 1.c \\
\hline $\begin{array}{l}\text { Estudio de } \\
\text { cohorte }\end{array}$ & 8 & $\begin{array}{l}\text { Maria C. Duggan, Li Wang, Jo } \\
\text { Ellen Wilson, Robert S. Dittus, } \\
\text { E. Wesley Ely, James C. } \\
\text { Jackson. }\end{array}$ & 2017 & USA & \begin{tabular}{|l|}
136 \\
pacientes \\
en \\
seguimient \\
0 \\
\end{tabular} & $\begin{array}{l}\text { Nivel } 3 . \\
\text { Grado } \\
\text { B }\end{array}$ \\
\hline
\end{tabular}




\begin{tabular}{|c|c|c|c|c|c|c|}
\hline $\begin{array}{l}\text { Estudio } \\
\text { observacional } \\
\text { multicéntrico }\end{array}$ & 7 & $\begin{array}{l}\text { M. Raurell-Torredà, S. Arias- } \\
\text { Rivera, et al. }\end{array}$ & 2018 & España & $86 \mathrm{UCI}$ & $\begin{array}{l}\text { Nivel } 3 \\
\text { Grado } \\
\text { B }\end{array}$ \\
\hline $\begin{array}{l}\text { Ensayo de } \\
\text { control } \\
\text { aleatorio }\end{array}$ & 9 & $\begin{array}{l}\text { George E. Sayde, Andrei } \\
\text { Stefanescub, Erich Conrad, } \\
\text { Nathan Nielsen, Rachel } \\
\text { Hammeruna }\end{array}$ & 2020 & USA & \begin{tabular}{|l|}
265 \\
pacientes
\end{tabular} & $\begin{array}{l}\text { Nivel 1 } \\
\text { C. } \\
\text { Grado } \\
\text { B }\end{array}$ \\
\hline $\begin{array}{l}\text { Cohorte } \\
\text { observacional }\end{array}$ & 7 & $\begin{array}{l}\text { Marcus T. Altman, Melissa P. } \\
\text { Knauert, Terrence E. Murphy, } \\
\text { et al }\end{array}$ & 2018 & USA & $\begin{array}{l}422 \\
\text { pacientes }\end{array}$ & $\begin{array}{l}\text { nivel } 3 . \\
\text { e. } \\
\text { Grado } \\
\text { B }\end{array}$ \\
\hline $\begin{array}{l}\text { Ensayo } \\
\text { controlado } \\
\text { aleatorizado }\end{array}$ & 9 & $\begin{array}{l}\text { Christina Jones, Carl Bäckman, } \\
\text { Maurizia Capuzzo, Ingrid } \\
\text { Egerod, Hans Flaatten, et al. }\end{array}$ & 2010 & \begin{tabular}{|l} 
Paises \\
Bajos
\end{tabular} & \begin{tabular}{|l|}
352 \\
pacientes
\end{tabular} & $\begin{array}{l}\text { Nivel } \\
\text { 1C. } \\
\text { Grado } \\
\text { A }\end{array}$ \\
\hline $\begin{array}{l}\text { Casos y } \\
\text { controles }\end{array}$ & 7 & $\begin{array}{l}\text { Jingjing Zhao, Li Yao, } \\
\text { Changqing Wang, Yun Sun } \\
\text { and Zhongwu Sun }\end{array}$ & 2015 & \begin{tabular}{|l|} 
Repúblic \\
a de \\
Corea \\
\end{tabular} & \begin{tabular}{|l|}
332 \\
pacientes
\end{tabular} & $\begin{array}{l}\text { Nivel } 3 \\
\text { d } \\
\text { Grado } \\
\text { A }\end{array}$ \\
\hline Ensayo clínico & 9 & \begin{tabular}{|l|} 
Jalil Azimian, \\
Omid Assar, \\
Amir Javadi \\
Zohreh Froughi \\
\end{tabular} & 2019 & Irán & \begin{tabular}{|l|}
68 \\
pacientes
\end{tabular} & $\begin{array}{l}\text { Nivel } \\
\text { 1C. } \\
\text { Grado } \\
\text { B } \\
\end{array}$ \\
\hline $\begin{array}{l}\text { Ensayo } \\
\text { aleatorizado }\end{array}$ & 8 & $\begin{array}{l}\text { J. Randall Curtis, Patsy D. } \\
\text { Treece, Elizabeth L. Nielsen, } \\
\text { Julia Gold, et al. }\end{array}$ & 2016 & USA & \begin{tabular}{|l|}
268 \\
familias
\end{tabular} & $\begin{array}{l}\text { Nivel 1 } \\
\text { c. } \\
\text { Grado } \\
\text { A }\end{array}$ \\
\hline $\begin{array}{l}\text { Ensayo } \\
\text { controlado } \\
\text { aleatorio }\end{array}$ & 8 & $\begin{array}{l}\text { By Paula L. Cairns } \\
\text { Harleah G. Buck } \\
\text { Kevin E. Kip } \\
\text { armen S. et al. } \\
\end{array}$ & 2019 & USA & \begin{tabular}{|l|}
10 \\
pacientes
\end{tabular} & $\begin{array}{l}\text { Nivel } 1 \\
\text { c. } \\
\text { Grado } \\
\text { A }\end{array}$ \\
\hline $\begin{array}{l}\text { Ensayo } \\
\text { controlado } \\
\text { aleatorio } \\
\text { multicéntrico }\end{array}$ & 8 & $\begin{array}{l}\text { James C. Jackson } \\
\text { Timothy D. Girard } \\
\text { Sharon M. E. Wesley Ely et al. }\end{array}$ & 2010 & Europa & $\begin{array}{l}187 \\
\text { pacientes }\end{array}$ & $\begin{array}{l}\text { Nivel } \\
1 \mathrm{~d} \\
\text { Grado } \\
\text { B }\end{array}$ \\
\hline $\begin{array}{l}\text { Estudio de } \\
\text { control } \\
\text { histórico }\end{array}$ & 8 & $\begin{array}{l}\text { Joanne McPeake } \\
\text { Martin Shaw } \\
\text { Theodore J. Iwashyna } \\
\text { Malcolm et al }\end{array}$ & 2017 & Europa & \begin{tabular}{l|}
40 \\
pacientes \\
seguimient \\
o a 12 \\
meses
\end{tabular} & $\begin{array}{l}\text { Nivel 2 } \\
\text { d. } \\
\text { Grado } \\
\text { B }\end{array}$ \\
\hline $\begin{array}{l}\text { Estudio } \\
\text { descriptivo } \\
\text { longitudinal, } \\
\text { prospectivo } \\
\end{array}$ & 7 & By Amy Petrinec, RN, $\mathrm{PhD}$ & 2017 & USA & $\begin{array}{l}30 \\
\text { participant } \\
\text { es }\end{array}$ & $\begin{array}{l}\text { Nivel } 4 \\
\text { Grado } \\
\text { B }\end{array}$ \\
\hline $\begin{array}{l}\text { Estudio de } \\
\text { cohorte } \\
\text { retrospectiva }\end{array}$ & 7 & $\begin{array}{l}\text { Barbara Riegel, Liming Huang, } \\
\text { Mark E. Mikkelsen, et al }\end{array}$ & 2018 & USA & \begin{tabular}{|l|}
21520 \\
sobrevivie \\
ntes
\end{tabular} & $\begin{array}{l}\text { Nive1 2 } \\
\text { d. } \\
\text { Grado } \\
\text { B }\end{array}$ \\
\hline Cohorte & 8 & $\begin{array}{l}\text { Edward Heydon, Bradley } \\
\text { Wibrow, Angela Jacques, } \\
\text { MBiostat et al. } \\
\end{array}$ & 2019 & Australia & \begin{tabular}{|l|}
50 \\
pacientes
\end{tabular} & $\begin{array}{l}\text { Nivel 3 } \\
\text { Grado } \\
\text { B } \\
\end{array}$ \\
\hline Cohorte & 9 & Yeon Jin Jeong \& Jiyeon Kang & 2019 & \begin{tabular}{|l|} 
Repúblic \\
a de \\
Corea
\end{tabular} & \begin{tabular}{|l|}
536 \\
sobrevivie \\
ntes
\end{tabular} & $\begin{array}{l}\text { Nivel } \\
\text { 1c. } \\
\text { Grado } \\
\text { A } \\
\end{array}$ \\
\hline
\end{tabular}

Fuente: Elaboración propia, datos revisión 2010 -2020

\section{Discusión}

A continuación, se presentan las categorías temáticas producto de los hallazgos de la literatura analizada.

\section{Aspecto cognitivo en el síndrome post UCI}

Las deficiencias cognitivas de reducción de la memoria y concentración ocurren en aproximadamente en el 25\% de los sobrevivientes y duran hasta 8 años (9). Seis de cada diez pacientes con deterioro cognitivo o discapacidad prexistente desarrollaron uno o más problemas de PICS (10). Precisamente, el deterioro cognitivo es el más común (11), dados ciertos factores de la UCI, como la estancia hospitalaria, aparición de delirium, uso de benzodiacepinas, ventilación mecánica prolongada (12) y las alteraciones del sueño (13). El paso por la UCI deteriora día a día a las personas, de acuerdo con la complejidad de su situación (14). Las principales secuelas del deterioro cognitivo global se presentan con disminución o afectación en las habilidades visoespaciales/constructivas, problemas de memoria inmediata y diferida, o la concentración. Se presentaron alteraciones en las habilidades propioceptivas, que involucran tareas motoras y manuales, entre los 3 y 12 meses del alta de UCI, con un impacto inmenso en la vida cotidiana de quienes las manifiestan (15) (16). El nivel de educación asociado con el logro ocupacional, mayores ingresos, mejores habilidades cognitivas, pensamiento crítico y redes de apoyo más amplias podrían representar mejores recursos para facilitar la recuperación (17). Similar labor facilitadora asumen las intervenciones preventivas de estimulación durante la internación en la UCI, como la escritura de diarios de UCI y disminución de sedación $(18,10)$. 


\section{Aspectos osteomusculares en el síndrome post UCI}

La fatiga abrumadora, disnea, debilidad, dolor, reducción de la actividad e incapacidad para volver a las actividades anteriores a la enfermedad crítica son factores que se describen con frecuencia en pacientes que estuvieron en UCI y pueden perdurar entre 1 y 5 años $(19,20)$. Una baja adherencia a la rehabilitación física disminuye el regreso a las actividades de estudio y laborales de los pacientes (21). La rehabilitación temprana y constante mejora la movilidad tras los primeros 3 meses del alta (22), brindando un mejor pronóstico en la recuperación a largo plazo de las capacidades motoras $(23,24,25)$.

\section{Aspectos psicoemocionales en el síndrome post UCI}

Durante el período inicial de estado crítico de la enfermedad, en una población post UCI, pueden originarse alternancias emocionales o episodios amnésicos. Estos conducen a la pérdida paulatina del recuerdo exacto de los hechos y a evocaciones delirantes, que se traducen en una retentiva falsa de la estancia en la UCI, causantes de síntomas relacionados con ansiedad y trastornos de estrés postraumático, TEPT $(26,27)$. También se identificó que la dificultad para dormir, el deterioro en la memoria y la falta de concentración son consecuencias que persisten luego de la salida, y ante una exposición a factores predisponentes (28). La disfunción ejecutiva puede resultar en deficiencias o declive funcional, desempleo, incumplimiento de medicación y deterioro de las relaciones sociales $(3,29)$. Aquellos pacientes que después de 6 meses del egreso no habían regresado a su trabajo tenían más probabilidades de tener una discapacidad, de moderada a grave, tras el alta de UCI, aumento de la disfunción psicológica y mala calidad de vida relacionada con la salud (30,31). La promoción de las habilidades de afrontamiento y la formación en mindfulnesssirven como herramientas para contrarrestar estas situaciones (32). Los enfermeros también potencian el apoyo a la salud mental de manera más efectiva en los pacientes, mientras estos permanecen en la UCI (33). De igual forma, el respaldo emocional y la presencia de familiares, amigos y del personal de salud, los factores ambientales como la televisión, las fotografías, las imágenes y la música, así como la fisioterapia y las caminatas generan emociones positivas durante la estancia en la UCI $(34,35)$.

\section{Estrategias de prevención}

Los pacientes que manifiestan el síndrome post UCI experimentan el sufrimiento al igual que las familias que luchan por su recuperación. Resulta muy importante que se trabaje en evitar la aparición de este síndrome; para esto, se proponen diversas estrategias de prevención, tales como la conciliación de medicamentos en el periodo posthospitalario temprano, dada la transición de alto riesgo que en pacientes complejos requiere un seguimiento más cercano (36). En la misma línea, el ambiente de la UCI destaca la necesidad de eliminar el ruido innecesario: revisar, silenciar y ajustar las alarmas de las bombas para minimizar la interrupción del sueño del paciente y evitar las conversaciones no clínicas en la estación de enfermeras o junto a la cama del paciente (37). Por su parte, la rehabilitación temprana y los centros de rehabilitación recogen unas de las principales estrategias de prevención del síndrome en diferentes etapas $(38,39)$.

La movilización precoz entre el segundo y quinto día permite disminuir la incidencia y las secuelas de la debilidad adquirida en las UCI $(40,41,42,43,44,45,46)$. Por tanto, los programas de apoyo entre pares de la UCI implican una interacción regular y constante con otros sobrevivientes de la UCI y sus familias (32). 


\section{Síndrome post unidad cuidado intensivo en la familia PICS-F}

Los familiares de los pacientes de la unidad de cuidados intensivos (UCI) corren el riesgo de enfrentar problemas psicológicos durante y después de la enfermedad en la UCI, tales como ansiedad, depresión, altos niveles de estrés relacionados con el afectación estimada en el pariente hospitalizado en UCI (35), así como TEPT y disminución de la calidad de vida $(47,48)$. En consecuencia, los familiares de pacientes post UCI presentan una disminución de la calidad de vida relacionada con la salud mental (50). En cuanto a los cuidadores, la calidad subjetiva de su sueño se correlaciona con síntomas depresivos y la carga de responsabilidades constantes a lo largo del tiempo (49). Los factores de riesgo identificados por el cuidador para PICS-F son género femenino, pariente más joven, ser cónyuge, edad de los pacientes mayores, condiciones más crónicas, interferencia en el estilo de vida, menor dominio y sobrecarga (42). Lastimosamente, las intervenciones efectivas para apoyar a los cuidadores durante la enfermedad crítica son limitadas, aunque se reportan intervenciones antiestrés por medio del Entrenamiento Enfocado en la Conciencia de las Sensaciones (E-ECS), el cual facilita la atención centrada en la familia (51). Los estudios de intervención que utilizan diarios de la UCI o estrategias de comunicación personalizadas han demostrado ser prometedores para disminuir la carga de los síntomas del TEPT (50). Así, el personal de enfermería puede desempeñar un papel fundamental en la satisfacción de las necesidades de la atención centrada en la familia, especialmente durante las diversas transiciones y transferencias que realizan los pacientes de la UCI (52).

\section{Herramientas para evaluar el PICS}

Los métodos utilizados para evaluar factores predisponentes, desarrollo, gravedad y consecuencias del síndrome, son variados, ya que se consideran diversos aspectos por separado. El primero mide los aspectos cognitivos mediante la Evaluación Cognitiva de Montreal (MoCA) y el soporte de tecnología robótica con las pruebas Trailmaking A y B (10,32,15). Un segundo tiene en cuenta los aspectos psicológicos con la aplicación del instrumento BSI-18 (53), que evalúa el malestar psicológico y las comorbilidades en los pacientes, identificando la somatización, depresión y ansiedad (26). El tercero o lista de verificación trastorno de estrés postraumático (PCL-5) busca establecer la gravedad de los síntomas del TEPT (53). El cuarto, de aspectos físicos, aplica el índice de Barthel y la escala del Consejo de Investigación Médica para la Fuerza Muscular $(10,42)$. El quinto se centra en calidad de vida, a través de la escala EuroQol-5 (EQ-5D-3L) (10), mientras que con la escala analógica visual (EQ-VAS) los pacientes puntúan subjetivamente su estado de salud entre 0 y 100 (53,26), y con la encuesta de salud general Medical Outcomes Study, versión 2 (SF-36v2) de formato corto con 36 ítems, se miden los componentes físicos y mentales de la Calidad de Vida Relacionada con la Salud, CVRS $(33,47,54)$.

Es necesario el desarrollo de una herramienta que logre evaluar o calcular el síndrome en el conjunto de sus aspectos (55). De este modo, el instrumento Post-Intensive Care Syndrome Questionnaire (PICSQ) es un cuestionario diseñado para crear esta herramienta que diagnostique el síndrome en los sobrevivientes de las unidades de cuidado intensivo y que cuente con propiedades psicométricas (56).

\section{Conclusiones}

El síndrome post unidad de cuidado intensivo es un fenómeno que presenta diversas características relacionadas con secuelas en la salud física y mental en los pacientes sobrevivientes de la UCI, lo que produce cambios es sus estilos de vida y cargas para sus familias. Es relevante contar con estrategias de prevención farmacológicas, como el manejo de los niveles de sedación, y no farmacológicas, como la calidad del sueño, prevención y detección del delirium, rehabilitación física de manera temprana durante la hospitalización, y 
seguimiento posterior al egreso. Aunque los estudios que realizan un diagnóstico sobre el síndrome utilizaron diferentes instrumentos, se evidenció la necesidad de contar con un medio unificado que mida el PICS de manera integral para obtener datos precisos. De forma similar, se requieren estudios que examinen si las discapacidades funcionales tempranas, identificadas en algunas investigaciones, evolucionan o permanecen después del periodo de seguimiento temprano. En la actualidad, existe evidencia mínima de intervenciones efectivas para apoyar a este grupo de pacientes después del alta hospitalaria.

\section{Referencias}

1. Inoue S, Hatakeyama J, Kondo Y, Hifumi T, Sakuramoto H, Kawasaki T, et al. Post-intensive care syndrome: its pathophysiology, prevention, and future directions. Acute Med Surg. 2019;6(3):233-46. Doi https://doi.org/1 $0.1002 / \mathrm{ams} 2.415$

2. Myers EA, Smith DA, Allen SR, Kaplan LJ. Post-ICU syndrome: rescuing the undiagnosed. J Am Acad Physician Assist. 2016;29(4):34-7. https://doi.org/10.1097/01.jaa.0000481401.21841.32

3. Tripathy S, Acharya SP, Singh S, Patra S, Mishra BR, Kar N. Post-traumatic stress symptoms, anxiety, and depression in patients after intensive care unit discharge- a longitudinal cohort study from a LMIC tertiary care centre. BMC Psychiatry. 2020;20(1):1-11. https://doi.org/10.1186/s12888-020-02632-x

4. Needham DM, Davidson J, Cohen H, Hopkins RO, Weinert C, Wunsch H, et al. Improving long-term outcomes after discharge from intensive care unit: report from a stakeholders' conference. Crit Care Med. 2012;40(2):502-9. https://doi.org/10.1097/ccm.0b013e318232da75

5. Marin T. Delirium (adults): risk screening, assessment and management. JBI Evid Sum. 2019;1-5. Disponible en http://ovidsp.dc1.ovid.com/sp-3.33.0b/ovidweb.cgi?\&S=HLGKFPPEPCACMCKKKPDKPGHOEKIK AA00\&Link+Set $=$ S.sh.21\%7csI_190

6. Peters MDJ, Godfrey C, McInerney P, Munn Z, Tricco AC, Khalil, H. Scoping reviews. En: Aromataris E, Munn Z, editores. JBI Manual for evidence synthesis. 2020. Cap. 11, Adelaida (Australia): JBI Global Wiki. Disponible en https://synthesismanual.jbi.global

7. Cabello JB. Lectura crítica de la evidencia clínica. Barcelona: Elsevier; 2015. 216 p.

8. Yépes-Núñez JJ, Urrutia G, Romero-García M, Alonso-Fernández S. Declaración Prisma 2020: una guía actualizada para la publicación de revisiones sistemáticas. Rev Española Cardiol. 2021;74(9):790-9. http://dx.doi.org/10.1 016/j.recesp.2021.06.016

9. Kang J, Jeong YJ. Embracing the new vulnerable self: a grounded theory approach on critical care survivors' postintensive care syndrome. Intensive Crit Care Nurs. 2018;49:44-50. https://doi.org/10.1016/j.iccn.2018.08.004

10. Kerckhoffs MC, Kosasi FFL, Soliman IW, van Delden JJM, Cremer OL, de Lange DW, et al. Determinants of self-reported unacceptable outcome of intensive care treatment 1 year after discharge. Intensive Care Med. 2019;45(6):806-14. https://doi.org/10.1007/s00134-019-05583-4

11. Marra A, Pandharipande PP, Girard TD, Patel MB, Hughes CG, Jackson JC, et al. Co-occurrence of post-intensive care syndrome problems among 406 survivors of critical illness. Crit Care Med. 2018;46(9):1393-401. https:// doi.org/10.1097/ccm.0000000000003218

12. Martins JB, dos Santos AA, de Macedo Júnior LJJ, Eberle CC. Avaliação da prevalência de delirium em uma unidade de terapia intensiva pública. Enferm em Foco. 2019;10(3):76-81. https://doi.org/10.21675/2357-707X.2019. V10.N3.1759

13. Altman MT, Knauert MP, Murphy TE, Ahasic AM, Chauhan Z, Pisani MA. Association of intensive care unit delirium with sleep disturbance and functional disability after critical illness: an observational cohort study. Ann Intensive Care. 2018;8(1):63. https://doi.org/10.1186/s13613-018-0408-4

14. Karnatovskaia LV, Schulte PJ, Philbrick KL, Johnson MM, Anderson BK, Gajic O, et al. Psychocognitive sequelae of critical illness and correlation with 3\#months follow up. J Crit Care. 2019;52:166-71. https://doi.org/10.1 $016 /$ j.jcrc.2019.04.028 
15. Wood MD, Maslove DM, Muscedere J, Scott SH, Boyd JG. Robotic technology provides objective and quantifiable metrics of neurocognitive functioning in survivors of critical illness: a feasibility study. J Crit Care. 2018;48:228-36. Disponible en https://doi.org/10.1016/j.jcrc.2018.09.011

16. Jackson JC, Girard TD, Gordon SM, Thompson JL, Shintani AK, Thomason JWW, et al. Long-term cognitive and psychological outcomes in the awakening and breathing controlled trial. Am J Respir Crit Care Med. 2010;182(2):183-91. https://doi.org/10.1164/rccm.200903-0442oc

17. Marra A, Pandharipande PP, Girard TD, Patel MB, Hughes CG, Jackson JC, et al. Co-occurrence of post-intensive care syndrome problems among 406 survivors of critical illness. Crit Care Med. 2018;46(9):1393-401. https:// doi.org/10.1097/ccm.0000000000003218

18. Zhao J, Yao L, Wang C, Sun Y, Sun Z. The effects of cognitive intervention on cognitive impairments after intensive care unit admission. Neuropsychol Rehabil. 2015;27(3):301-17. https://doi.org/10.1080/09602011.2015.107 8246

19. Thurston LM, Milnes SL, Hodgson CL, Berkovic DE, Ayton DR, Iwashyna TJ, et al. Defining patient-centered recovery after critical illness - a qualitative study. J Crit Care. 2020;57:84-90. https://doi.org/10.1016/j.jcrc.2 020.01 .028

20. Rai S, Anthony L, Needham DM, Georgousopoulou EN, Sudheer B, Brown R, et al. Barriers to rehabilitation after critical illness: a survey of multidisciplinary healthcare professionals caring for ICU survivors in an acute care hospital. Aust Crit Care. 2019;33(3):264-71. https://doi.org/10.1016/j.aucc.2019.05.006

21. das Neves AV., Vásquez DN, Loudet CI, Intile D, Sáenz MG, Marchena C, et al. Symptom burden and healthrelated quality of life among intensive care unit survivors in Argentina: a prospective cohort study. J Crit Care. 2015;30(5):1049-54. Disponible en https://docksci.com/symptom-burden-and-health-related-quality-of-life-a mong-intensive-care-unit-surv_5a3cd71ed64ab25bb20bfa8f.html

22. Sidiras G, Patsaki I, Karatzanos E, Dakoutrou M, Kouvarakos A, Mitsiou G, et al. Long term follow-up of quality of life and functional ability in patients with ICU acquired weakness - a post hoc analysis. J Crit Care. 2019;53:223-30. https://doi.org/10.1016/j.jcrc.2019.06.022

23. Seo YJ, Park SR, Lee JH, Jung C, Choi KH, Hong SK, et al. Feasibility, safety, and functional recovery after active rehabilitation in critically ill surgical patients. Aust Crit Care. 2020;33(3):281-6. https://doi.org/10.1016/j.au cc.2019.07.005

24. Patsaki I, Gerovasili V, Sidiras G, Karatzanos E, Mitsiou G, Papadopoulos E, et al. Effect of neuromuscular stimulation and individualized rehabilitation on muscle strength in intensive care unit survivors: a randomized trial. J Crit Care. 2017;40:76-82. https://doi.org/10.1016/j.jcrc.2017.03.014

25. Riegel B, Huang L, Mikkelsen ME, Kutney-Lee A, Hanlon AL, Murtaugh CM, et al. Early post-intensive care syndrome among older adult sepsis survivors receiving home care. J Am Geriatr Soc. 2018;67(3):520-6. https: //doi.org/10.1111/jgs.15691

26. Vlake JH, van Genderen ME, Schut A, Verkade M, Wils EJ, Gommers D, et al. Patients suffering from psychological impairments following critical illness are in need of information. J Intensive Care. 2020;8(1):1-10. https://doi .org/10.1186/s40560-019-0422-0

27. Janssen DGA, Jonker J, van Dijk D, Heerdink ER, Egberts TCG, Slooter AJC. Psychotropic medication use in former ICU patients with mental health problems: a prospective observational follow-up study. J Crit Care. 2020;59:112-7. https://doi.org/10.1016/j.jcrc.2020.06.002

28. Azimian J, Assar O, Javadi A, Froughi Z. Effect of using eye masks and earplugs on the risk of post-traumatic stress disorder development in patients admitted to cardiac surgery intensive care units. Indian J Crit Care Med. 2019;23(1):31-4. https://doi.org/10.5005/jp-journals-10071-23109

29. Duggan MC, Wang L, Wilson JE, Dittus RS, Ely EW, Jackson JC. The relationship between executive dysfunction, depression, and mental health-related quality of life in survivors of critical illness: results from the BRAIN-ICU investigation. J Crit Care. 2017;37:72-9. https://doi.org/10.1016/j.jcrc.2016.08.023

30. Hodgson CL, Haines KJ, Bailey M, Barrett J, Bellomo R, Bucknall T, et al. Predictors of return to work in survivors of critical illness. J Crit Care. 2018;48:21-5. https://doi.org/10.1016/j.jcrc.2018.08.005 
31. Detsky ME, Kohn R, Delman AM, Buehler AE, Kent SA, Ciuffetelli IV, et al. Patients' perceptions and ICU clinicians predictions of quality of life following critical illness. J Crit Care. 2018;48:352-6. https://doi.org/10 $.1016 /$ j.jcrc.2018.09.034

32. Sevin CM, Bloom SL, Jackson JC, Wang L, Wesley Ely E, Stollings JL. Comprehensive care of ICU survivors: development and implementation of an ICU recovery center. J Crit Care. 2018;46:141-8. https://doi.org/10. 1016/j.jcrc.2018.02.011

33. Heydon E, Wibrow B, Jacques A, Sonawane R, Anstey M. The needs of patients with post-intensive care syndrome: a prospective, observational study. Aust Crit Care. 2019;33(2):116-22. https://doi.org/10.1016/j.aucc.2019.0 4.002

34. van Mol M, Ista E, van Dijk M. Implementation and evaluation of a follow-up programme after intensive care treatment: a practice development project. Intensive Crit Care Nurs. 2018;49:6-13. https://doi.org/10.1016/j .iccn.2018.04.009

35. Dziadzko V, Dziadzko MA, Johnson MM, Gajic O, Karnatovskaia LV. Acute psychological trauma in the critically ill: patient and family perspectives. Gen Hosp Psychiatry. 2017;47:68-74. https://doi.org/10.1016/j.genhospp sych.2017.04.009

36. Stollings JL, Bloom SL, Wang L, Ely EW, Jackson JC, Sevin CM. Critical care pharmacists and medication management in an ICU recovery center. Ann Pharmacother. 2018;52(8):713-23. https://doi.org/10.1177/10 60028018759343

37. Tronstad O, Flaws D, Lye I, Fraser JF, Patterson S. The intensive care unit environment from the perspective of medical, allied health and nursing clinicians: a qualitative study to inform design of the 'ideal' bedspace. Aust Crit Care. 202;34(1):15-22. https://doi.org/10.1016/j.aucc.2020.06.003

38. Chiarici A, Andrenelli E, Serpilli O, Andreolini M, Tedesco S, Pomponio G, et al. An early tailored approach is the key to effective rehabilitation in the intensive care unit. Arch Phys Med Rehabil. 2019;100(8):1506-14. htt ps://doi.org/10.1016/j.apmr.2019.01.015

39. Sayde GE, Stefanescu A, Conrad E, Nielsen N, Hammer R. Implementing an intensive care unit (ICU) diary program at a large academic medical center: results from a randomized control trial evaluating psychological morbidity associated with critical illness. Gen Hosp Psychiatry. 2020;66:96-102. https://doi.org/10.1016/j.ge nhosppsych.2020.06.017

40. Raurell-Torredà M, Arias-Rivera S, Martí JD, Frade-Mera MJ, Zaragoza-García I, Gallart E, et al. Degree of implementation of preventive strategies for post-ICU syndrome: multi-centre, observational study in Spain. Enferm Intensiva. 2019;30(2):59-71. https://doi.org/10.1016/j.enfi.2018.04.004

41. McPeake J, Shaw M, Iwashyna TJ, Daniel M, Devine H, Jarvie L, et al. Intensive care syndrome: promoting independence and return to employment (InS:PIRE). Early evaluation of a complex intervention. PLoS One. 2017;12(11):1-13. https://doi.org/10.1371/journal.pone.0188028

42. Torres J, Carvalho D, Molinos E, Vales C, Ferreira A, Dias CC, et al. The impact of the patient post-intensive care syndrome components upon caregiver burden. Med Intensiva. 2017;41(8):454-60. https://doi.org/10.1016/j. medin.2016.12.005

43. van Beusekom I, Bakhshi-Raiez F, de Keizer NF, van der Schaaf M, Termorshuizen F, Dongelmans DA. Dutch ICU survivors have more consultations with general practitioners before and after ICU admission compared to a matched control group from the general population. PLoS One. 2019;14(5):1-13. https://doi.org/10.1371/ journal.pone.0217225

44. van Beusekom I, Bakhshi-Raiez F, van der Schaaf M, Dongelmans DA, Busschers WB, de Keizer NF. The influence of clinical variables on the risk of developing chronic conditions in ICU survivors. J Crit Care. 2020;55:134-9. https://doi.org/10.1016/j.jcrc.2019.10.014

45. Hanifa ALB, Glæemose AO, Laursen BS. Picking up the pieces: qualitative evaluation of follow-up consultations post intensive care admission. Intensive Crit Care Nurs. 2018;48:85-91. https://doi.org/10.1016/j.iccn.2018.0 6.004 
46. Stollings JL, Bloom SL, Wang L, Ely EW, Jackson JC, Sevin CM. Critical care pharmacists and medication management in an ICU recovery center. Ann Pharmacother. 2018;52(8):713-23. https://doi.org/10.1177/10 60028018759343

47. Petrinec A. Post-intensive care syndrome in family decision makers of long-term acute care hospital patients. Am J Crit Care. 2017;26(5):416-22. https://doi.org/10.4037/ajcc2017414

48. Lewis CL, Taylor JZ. Impact of prior ICU experience on ICU patient family members' psychological distress: a descriptive study. Intensive Crit Care Nurs. 2017;43:129-35. https://doi.org/10.1016/j.iccn.2017.09.008

49. Choi JY, Tate JA, Donahoe MP, Ren D, Hoffman LA, Chasens ER. Sleep in family caregivers of ICU survivors for two months post-ICU discharge. Intensive Crit Care Nurs. 2016;37:11-18. https://doi.org/10.1016/j.iccn .2016.07.003

50. Petrinec AB, Martin BR. Post-intensive care syndrome symptoms and health-related quality of life in family decision-makers of critically ill patients. Palliat Support Care. 2018;16(6):719-24. https://doi.org/10.1017/s1 478951517001043

51. Cairns PL, Buck HG, Kip KE, Rodríguez CS, Liang Z, Munro CL. Stress management intervention to prevent post-intensive care syndrome-family in patients' spouses. Am J Crit Care. 2019;28(6):471-7. https://doi.org/1 $0.4037 /$ ajcc2019668

52. Op ' $t$ Hoog SAJJ, Dautzenberg M, Eskes AM, Vermeulen H, Vloet LCM. The experiences and needs of relatives of intensive care unit patients during the transition from the intensive care unit to a general ward: a qualitative study. Aust Crit Care. 2020;33(6):526-32. https://doi.org/10.1016/j.aucc.2020.01.004

53. Rosendahl J, Kisyova H, Gawlytta R, Scherag A. Comparative validation of three screening instruments for posttraumatic stress disorder after intensive care. J Crit Care. 2019;53:149-54. https://doi.org/10.1016/j.jcrc.2 019.06 .016

54. Ferrand N, Zaouter C, Chastel B, Faye K, Fleureau C, Roze H, et al. Health related quality of life and predictive factors six months after intensive care unit discharge. Anaesth Crit Care Pain Med. 2019;38(2):137-41. https:/ /doi.org/10.1016/j.accpm.2018.05.007

55. Jeong YJ, Kang J. Development and validation of a questionnaire to measure post-intensive care syndrome. Intensive Crit Care Nurs. 2019;55:102756. https://doi.org/10.1016/j.iccn.2019.102756

56. Wang S, Allen D, Perkins A, Monahan P, Khan S, Lasiter S, et al. Validation of a new clinical tool for post-intensive care syndrome. Am J Crit Care. 2019;28(1):10-8. https://dx.doi.org/10.4037\%2Fajcc2019639

\section{Notas}

* $\quad$ Artículo de revisión integrativa.

\section{Licencia Creative Commons CC BY 4.0}

Limitaciones: El desconocimiento de la lengua china limitó la labor de la investigación, dado que se encontraron algunos estudios en este idioma.

Financiamiento: Ninguno.

Conflictos de interés: Los autores declaran no tener conflicto de intereses.

Cómo citar este artículo: Henao-Castaño A. M., Villamil Buitrago A. V., Marín Ramírez S., Cogollo Hernández C. A. Características del síndrome post cuidado intensivo: revisión de alcance. Investigación en Enfermería, Imagen y Desarrollo. 2021;23. https://doi.org/10.11144/Javeriana.ie23.csci 